\title{
Research on the Influence of Industrial Structure Adjustment on the Efficiency of Low Carbon Logistics in Hebei Province
}

\author{
Xiao Zhang \\ School of Economics and Management \\ Hebei University of Science and Technology \\ Shijiazhuang, Hebei, China
}

\begin{abstract}
Within the context of low carbonization, the adjustment and optimization of industrial structure plays an important role in improving the efficiency of low-carbon development of logistics industry in Hebei Province. Seven indicators including logistics carbon emissions, logistics industry gross production value and fixed asset investment are selected. The DEA and Tobit models are used to calculate the data of Hebei Province from 2006 to 2015. The results show that the overall efficiency of low carbon logistics in Hebei Province is not high. The efficiency rates of the six years are positive; and the adjustment of industrial structure has a positive effect on the efficiency of low-carbon logistics.
\end{abstract}

Keywords-Industrial Structure Adjustment; Low Carbon Logistics Efficiency; DEA Method; Tobit Model

\section{INTRODUCTION}

With the exhaustion of resources and the deterioration of natural environment, in order to seek sustainable economic development, low-carbon economy emerged as the times require. Its essence is to reduce $\mathrm{CO} 2$ emissions through technological innovation, industrial restructuring and new energy development. Logistics industry as a high energy consumption industry, reducing its carbon emissions is the key to achieve low-carbon development of the national economy, and the development of low-carbon logistics industry is also in a key position in the development of low-carbon economy. At the same time, the adjustment of industrial structure is closely linked with the development of low-carbon logistics. The government emphasizes the adjustment of industrial structure and the transformation of the mode of economic development. In this situation, the development of low-carbon logistics is an inevitable result. At the same time, the logistics industry plays a leading role in the tertiary industry, and the adjustment of industrial structure is closely linked with the development of low-carbon logistics. Low-carbon logistics takes low energy consumption, low pollution and low emissions as the main objectives in the logistics process, and then improves the utilization rate of logistics resources. Therefore, this paper constructs a low-carbon logistics efficiency evaluation model, studies the relationship between industrial restructuring and low-carbon logistics efficiency, in order to provide a theoretical basis for the government to adjust the industrial structure and promote the development of low-carbon logistics industry.

\section{LiterATURE REVIEW AT HOME AND ABROAD}

There are many studies on low-carbon logistics and adjustment of industrial results by domestic and foreign scholars. For the study of industrial structure adjustment, Zhang Yayu and Song Gang used grey relational analysis method to calculate the industrial structure and logistics demand of Guangxi and Hunan provinces respectively; Liu Hao [3] analyzed the industrial structure and main characteristics of Tibet region, and discussed Tibet industry from two aspects of time series evolution law and spatial change. The relationship between structural optimization and regional logistics development; Zou Xiao [4] used the principal component analysis method to measure the level of interaction between industrial structure optimization and regional logistics capacity in Hunan Province.

For the study of low-carbon logistics, Hickman [5-6] and others studied the optimization of logistics transportation, distribution and logistics network in low-carbon economy. Li Yonglin [7] introduced carbon emissions from logistics industry and GDP of logistics industry into the evaluation system of low-carbon logistics efficiency as input and output indicators respectively, and based on DEA model, 31 provinces in China (self-government) Empirical analysis of low-carbon logistics efficiency in autonomous regions and municipalities directly under the Central Government. Lu Juanjuan [8] established an evaluation index system of low-carbon logistics efficiency and used DEA data envelopment analysis to evaluate the efficiency of low-carbon logistics in Anhui Province and cities. Cao Bingbin [9] establishes a low-carbon logistics efficiency evaluation based on the perspective of low-carbon development. Tang Zhongming builds a logistics efficiency evaluation system under low-carbon economy from two perspectives of internal efficiency and external efficiency. Based on data envelopment analysis (DEA), an evaluation model of urban logistics efficiency is established to make an empirical study of Wuhan urban agglomeration. 


\section{EVAluAtion AND ANALYSIS OF LOW CARBON LOGISTICS EFFICIENCY}

\section{A. Data envelopment analysis}

Data Envelopment Analysis (DEA) is an analysis method to measure the efficiency combination of input and output. It was first proposed by Charnes et al. in the late 1970s. The common models of DEA are CCR and BCC models. The following is an introduction to the principle of the model.

Assuming the evaluation of low-carbon logistics efficiency in a region in K-year, input and output indicators are $\mathrm{L}$ and $\mathrm{M}$ respectively, $x_{i j}$ represents the input of type resources in j-year, $y_{m j}$ represents the output of type resources in j-year. For the nth year of low carbon logistics in the region, the model is:

In the formula, theta is the technical efficiency index, lambda $\mathrm{j}$ is the weight variable, $s^{-}$and $s^{+}$are the relaxation variable and residual variable, $\varepsilon$ is the non-Archimedean infinitesimal, $\alpha_{1}^{T}$ and $\alpha_{2}^{T}$ dimension $\mathrm{m}$ dimension and $\mathrm{K}$ dimension unit vector.

$$
\left\{\begin{array}{c}
\min \left(\theta-\varepsilon\left(\alpha_{1}^{T} s^{-}+\alpha_{2}^{T} s^{+}\right)\right) \\
\sum_{j=1}^{K} x_{i j} \lambda_{j}+s^{-}=\theta x_{i}^{n}, i=1,2, \cdots L \\
\sum_{j=1}^{K} x_{m j} \lambda_{j}-s^{+}=\theta y_{m}^{n}, m=1,2, \cdots M \\
\lambda_{j} \geq 0, j=1,2, \cdots K
\end{array}\right.
$$

B. Selection of evaluation index and data collection and collation

Based on the research of existing scholars, the three departments of transportation, warehousing and postal industry are defined as logistics industry. Under the low-carbon background, this paper takes the gross product of logistics industry, investment in fixed assets, employees, total length of transportation, freight volume and turnover as input indicators, and carbon emissions of logistics industry as output indicators [10] The measurement of carbon emissions is based on the total energy consumption of logistics industry. The indicators selected in this paper are as follows:

TABLE I INPUT-OUTPUT INDICATORS OF LOW CARBON LOGISTICS

\begin{tabular}{ll}
\hline Index type & Index \\
\hline & Gross Product $\left(x_{1}\right)$ \\
& Investment in Fixed Assets $\left(x_{2}\right)$ \\
& Employed Persons $\left(x_{3}\right)$ \\
Input indicators & Total Length of Highways $\left(x_{4}\right)$ \\
& Freight Traffic $\left(x_{5}\right)$ \\
& Ton-Kilometers $\left(x_{6}\right)$ \\
\hline Output index & Carbon dioxide emissions $(y)$ \\
\hline
\end{tabular}

This paper evaluates the logistics efficiency of Hebei Province horizontally and vertically with the classical DEA model, and calculates the relevant data of logistics industry in
Hebei Province from 2006 to 2015. It regards every year from 2006 to 2015 as a decision-making unit. The data mainly come from Hebei Economic Yearbook and Hebei National Economic and Social Development Statistical Bulletin.

\section{Efficiency evaluation and result analysis}

In this paper, data envelopment analysis method is used to calculate the relevant data of logistics industry in Hebei Province. The output and input data are imported to calculate the efficiency value of low-carbon logistics in 2006-2015. The results are shown in the table below.

\begin{tabular}{lllll} 
TABLE II & \multicolumn{5}{c}{ VALUE OF LOW CARBON LOGISTICS EFFICIENCY IN HEBEI } \\
& \multicolumn{5}{c}{ PROVINCE FROM 2006 TO 2015 } \\
\hline DUM & Crste & Vrste & Scale & Scale income \\
\hline 2006 & 1.00 & 1.00 & 1.00 & - \\
2007 & 1.00 & 1.00 & 1.00 & - \\
2008 & 1.00 & 1.00 & 1.00 & - \\
2009 & 0.80 & 0.99 & 0.81 & irs \\
2010 & 0.95 & 0.99 & 0.96 & irs \\
2011 & 1.00 & 1.00 & 1.00 & - \\
2012 & 1.00 & 1.00 & 1.00 & drs \\
2013 & 1.00 & 1.00 & 1.00 & - \\
2014 & 0.94 & 0.98 & 0.96 & drs \\
2015 & 0.97 & 1.00 & 0.97 & drs \\
Mean & 0.97 & 1.00 & 0.97 & \\
\hline
\end{tabular}

The values of Crste, Vrste and Scale calculated by DEA model show that the low-carbon logistics efficiency in Hebei Province has the following characteristics:

Overall, the average efficiency, average pure technical efficiency and average scale efficiency of low-carbon logistics industry in China have reached more than $90 \%$. The overall efficiency is general. The technical efficiency of low-carbon logistics in 2006, 2007 and 2008 is 1, which shows the development of low-carbon logistics efficiency in Hebei Province during the six years. Relatively good, has its development advantages, input redundancy and insufficient output. By comparison, the technical efficiency scores in the four years of 2009 and 2010 did not reach 1, especially in 2009, when the score was the lowest, indicating that the impact of the 2008 economic crisis was greater, making the economic development and low-carbon logistics efficiency in 2009 the most obvious impact.

The overall level of pure technical efficiency is relatively high. Only in 2010 and 2014, its score did not reach 1 . In the other years, the pure technical efficiency value was 1 . This shows that the advantages of pure technical efficiency in the development of low-carbon logistics in Hebei Province have been brought into full play. The pure technical efficiency value reaches 1 year, which indicates that the pure technical efficiency has been maintained in an effective state throughout the research period. Hebei Province has its own advantages in technology and management, and can gain benefits from investing all kinds of resources.

The improvement of scale efficiency can effectively promote the development of low-carbon logistics industry efficiency in Hebei Province. The scale efficiency reached 97\% 
in 2006-2015. From the tables and graphs, it can be seen that the overall score of technical efficiency is mainly affected by scale efficiency. The scale efficiency scores in 2009, 2010, 2014 and 2015 are not up to 1. This shows that the development of low-carbon logistics in Hebei Province has not reached the rational allocation of resources in these years, and needs to be improved. In the next time, adjust and optimize the input variables, so that the development efficiency of low-carbon logistics in Hebei Province can achieve better growth.

\section{EMPIRICAL ANALYSIS OF LOW CARBON LOGISTICS EFFICIENCY IN HEBEI PROVINCE BY INDUSTRIAL STRUCTURE ADJUSTMENT}

\section{A. Tobit regression model and index selection}

Tobit regression model, also known as sample selection model and constrained dependent variable model, is a model in which dependent variables satisfy certain constraints. This model was first proposed by Tobin. Its principle is as follows:

The expression of the model is as follows: $Z_{i}^{*}=\beta_{0}+$ $\beta^{T} X_{i}+c_{i}, i=1,2, \cdots, N$

$$
Z_{i}=\left\{\begin{array}{c}
Z_{i}^{*}, \text { if } Z_{i}^{*}>0 \\
0, \text { if } Z_{i}^{*} \leq 0
\end{array}\right.
$$

$Z_{i}^{*}$ is defined as dependent variable, $Z_{i}$ as efficiency value, $X_{i}$ as independent variable, $\beta^{T}$ as regression parameter vector, and $c_{i}$ as random error term. In this paper, the dependent variable is the efficiency value of low-carbon logistics in Hebei Province, and the efficiency value is more than 0 offline value, so it is more accurate to estimate Tobit model by maximum likelihood method.

There are two kinds of independent variables for industrial restructuring: (1) the proportion of the number of employees in the primary industry to the total number of employed people and the proportion of the output value of the primary industry to the gross domestic product; the smaller the two ratios, the faster the industrial restructuring is, the higher the degree of structural optimization is; (2) the second industry. The ratio of output value of industry and tertiary industry to output value of primary industry is the index of industrial structure. The higher the value, the faster the adjustment of industrial structure is, the better the structure is. Based on the current situation of Hebei Province and the existing literature, this paper takes the ratio of the added value of the secondary industry and the tertiary industry to the GDP as an independent variable, and takes the data from 2006 to 2015 as the model analysis.

\section{B. Empirical analysis}

Taking $X_{1}$ as independent variable and technical efficiency as dependent variable, the Tobit model is established. The Tobit model is regressed by Eviews. The results are shown in the table below. The model equation is as follows: $Z_{i}=\beta_{0}+\beta_{1} X_{i}+c, i=2006,2007, \cdots, 2015$.

TABLE III TOBIT MODEL REGRESSION RESULTS TABLE

\begin{tabular}{lllll}
\hline Variable & Coefficient & Std. Error & t-Statistic & Prob. \\
\hline $\mathrm{c}$ & -0.72237 & 0.4822 & -1.49794 & 0.1725 \\
$\mathrm{X}$ & 1.62836 & 0.4229 & 3.850492 & $0.0049 * * *$ \\
\hline \multicolumn{5}{c}{ Note: In the table , $* *, * * *^{*}$ are the significant levels of $10 \%, 5 \%$ and $1 \%}$.
\end{tabular}

From the table, we can see that the coefficient of independent variable is 1.62836 , and it is positively correlated with low-carbon logistics efficiency at a significant level of $1 \%$. This shows that the ratio of added value of secondary industry and added value of tertiary industry to GDP increases by $1 \%$, and the efficiency value of low-carbon logistics in Hebei Province will increase by $1.62836 \%$, which means that the adjustment of industrial structure is low. Carbon logistics efficiency is related, and can promote the growth of low-carbon logistics efficiency.

\section{CONCLUSION AND SUGGESTION}

This paper uses DEA to calculate and analyze the data of industrial restructuring and low-carbon logistics efficiency in Hebei Province from 2006 to 2015, studies the relationship between them, and draws the conclusion that the technical efficiency value of Hebei Province has reached an effective level in six years, but the scale efficiency will decrease by 2012-2015, which shows that Hebei Province is developing at the same time. It is also expanding the development of logistics industry, but when expanding the scale to promote development, there are problems of resource allocation such as insufficient manpower, material resources and funds; and the adjustment of industrial structure has a positive role in promoting the efficiency of low-carbon logistics in Hebei Province.

\section{REFERENCES}

[1] Zhang Yayu, Wei Wenjing. Empirical Study on the Relationship between Industrial Structure and Logistics Demand Based on Grey Theory [J].Logistics Technology, 2018,37(08):47-51.

[2] Song Gang, Feng Ru. Study on the Harmonious Development Model of Liaoning Port Logistics and Industrial Structure [J].2017(05):23-25.

[3] Liu Hao. Research on the optimization of industrial structure and regional logistics development in Tibet [J]. Journal of Tibet University for Nationalities (Philosophy and Social Sciences Edition),2018,39(03):76-82+155.

[4] Zou Xiao, Li Yuqin, Yang Dafa. Interactive comprehensive evaluation of industrial structure optimization and regional logistics capability in Hunan Province $[\mathrm{J}]$. Practice and understanding of mathematics, 2017,47(22):291-299.

[5] Hickman R, Ashiru. Banister. Transport and climate change:Simulating the options for carbon reduction in London[J].Transport Policy,2010,17(2):110 - 125

[6] Chains Environmental Imperative or Wasteful Distraction[J]International Journal of Physical Distribution\& Logistics Management,2010,40 (1) :42 - 60.

[7] Li Yonglin. Research on low-carbon logistics efficiency in China based on DEA [J], 2014(27): 23-25.

[8] Lu Juanjuan. Empirical study on low-carbon logistics efficiency of regional cities in Anhui Province [J]. Entrepreneur Tiandi (second half of the monthly), 2014 (07): 30-32.

[9] Cao Bingbin, Liujian. Evaluation Research on Urban Logistics Efficiency Based on Low Carbon Development - Taking Jiujiang City as an Example [J]. Logistics Science and Technology, 2016,39 (08): 9-11.

[10] Liujian, Tujian. Study on the Impact of China's Industrial Structure Adjustment on Low Carbon Logistics Efficiency: Empirical Analysis Based on Super Efficiency DEA Low Carbon Logistics Efficiency Evaluation Model [J]. Price Theory and Practice, 2017 (12): 130-133. 